\title{
COMMENT
}

Check for updates

\section{How neuroscience labs can limit their environmental impact}

Joseph D. Zak (10 ${ }^{1,2,4}$, Jenelle Wallace $\mathbb{1}^{1,2,3,4}$ and Venkatesh N. Murthy $\mathbb{1}^{1,2 凶}$

Neuroscience laboratories can take steps to 'go green' in a number of ways, including curbing unnecessary energy usage and reducing plastic waste. Such measures often rely on behavioural changes but need not affect scientific output.

Climate change and global warming are rapidly coming to a head. By current estimates, reported by the United Nations Intergovernmental Panel on Climate Change, we will need to curtail carbon emissions by a staggering $40 \%$ to $70 \%$ in the immediate term to prevent the runaway warming of our planet. Other evidence shows that a global disaster is already unfolding in the form of melting ice caps in polar regions of the planet ${ }^{1}$. This unfolding crisis will impact many aspects of our lives, including scientific output across all domains and disciplines. This much is clear: we must immediately change our energy consumption, resource management and collective behaviour to prevent the catastrophic destruction of our environment.

Are neuroscientists doing enough to curtail our contribution to the looming crisis? Social media platforms now connect us as scientists in a truly global community and host recurring discussions about how to limit our environmental impact. Much of the ongoing discourse is focused on direct efforts to reduce carbon emissions - for example, limiting conference travel or holding remote, video-linked seminar series. Although taking these steps until recently seemed optional to many of us, the COVID-19 pandemic has forced scientists around the world to give them a try. Hopefully, these experiences will demonstrate the personal, logistical and environmental advantages of adopting remote options more widely. Less travel means more time at the bench, with students and at home with family. The challenge will be to continue to normalize and implement these options after universities have reopened and flights have resumed. There are, however, even more everyday measures that can be undertaken to bolster these efforts, with cumulative effects that amount to more than a skipped flight. Importantly, these efforts require contributions from all members of a research group, not just those for whom travel is routine.

Our research spaces are vibrant communities that bring together people, ideas and resources; however, our research spaces also account for a disproportionate impact on energy consumption, with respect to other university functions. For instance, at Harvard University, research laboratories make up approximately $20 \%$ of the total building space, while accounting for nearly $44 \%$ of university-wide energy consumption (Harvard University Green Labs Program). Given our high energy consumption, it is obvious that we should work to reduce unnecessary usage. Here we argue for effective measures to reduce the energy consumption and limit the production of unnecessary waste materials in our laboratories. We also highlight initiatives of Harvard's Green Labs Program, which is part of the Harvard Office for Sustainability, as well as our experience participating in a friendly competition designed to encourage behavioural change.

Exhaust from fume hoods accounts for up to nearly half of the total energy consumption from research laboratories ${ }^{2}$. Therefore, one of the most basic efforts, resulting in nearly zero effect on research productivity, is to simply close the hood when not in use. Unfortunately, this seems to be more easily said than done - as was certainly true for our research group. Given our less-than-stellar conservation efforts, we were approached by the Harvard Green Labs Program to participate in their 'Shut the Sash' competition. The goal of the initiative is simple: reduce laboratory energy consumption by eliminating excess fume-hood exhaust through friendly competition between neighbouring research groups.

How does the competition programme work? Each lab is assigned a customized goal based on the number of hoods in each research space, historical usage patterns and air-flow exhaust ranges. Together, these factors allow for comparisons between research groups with differences in research habits and laboratory configurations. From each laboratory, real-time automated measurements of fume-hood exhaust are collected multiple times per hour and used to track performance on time scales ranging from days to months. Several times a year, winners are announced based on their reduction in fume-hood exhaust relative to baseline usage. Although the incentive to win the contest is modest - typically a sponsored lab lunch or happy hour - the friendly competition drives active engagement in many labs, where now over 20 labs across several departments at Harvard have participated in the programme. 
The positive and tangible outcomes of the programme are clear. In the initial 5 years of the programme, a 30\% reduction in university-wide fume-hood exhaust levels was achieved, which amounts to an annual reduction exceeding 300 metric tonnes of greenhouse gas emissions - the equivalent of the carbon footprint of 300 transcontinental flights across North America. Importantly, this programme provides a direct and impactful mechanism for all members of a research group to actively contribute to conservation efforts that reduce the environmental impact of laboratory research.

In our research group, we began each of our weekly group meetings with a reminder that fume hoods need to be shut when not in use and that each group member should 'shut the sash' whenever an opened one was encountered. Stickers reminding users about the potential for energy savings were placed on all fume-hood sashes. We found these reminders, accompanied by the ongoing friendly competition, to be a major driver of behavioural change that ultimately became a matter of habit and shaped our lab culture with respect to resource management. Through our work with the Green Labs Program, we dramatically reduced our laboratory energy consumption, a behaviour that we have maintained in the years following our participation in the competition.

Whereas shutting fume-hood sashes is unlikely to impact research output, there are other strategies that may improve environmental friendliness as well as lab organization and efficiency. Our lab uses an inventory management and ordering software that keeps track of our purchases, ensuring that perishable materials are not re-ordered by multiple lab members and reducing wasteful ordering. The system also keeps a record of all materials used in experiments and allows new lab members to quickly re-order the same supplies that were used in past experiments, improving reproducibility. In the future, we plan to add tags to orders indicating which are urgent and which can wait until more orders accrue, since bundling orders can improve shipping efficiency (and reduce costs for the lab)

What else can be done to limit the environmental impact of laboratory research? Other simple efforts can include turning off equipment and computer monitors when not in use, and defrosting freezers regularly to prevent ice buildup and maintain efficiency (probably also improving freezer lifetimes). Ultra-low-temperature freezers can be operated at $-70^{\circ} \mathrm{C}$ rather than $-80^{\circ} \mathrm{C}$, which can save energy while having no impact on biological samples ${ }^{3}$. Importantly, these simple efforts scale regardless of research group size, or department or university organization.

Some efforts to reduce energy consumption and waste in the laboratory may not contribute to scientific output and may require additional commitment. For example, laboratory research faces an escalating problem of single-use plastics and packaging waste accumulation. In many ways, we have crept towards prioritizing convenience and on-demand consumption over responsible resource management. Labs can consider switching to autoclavable, reusable materials, although it is important to remember that these typically do not provide net carbon savings unless they are re-used many times ${ }^{4}$. This may make sense for labs repeating certain tasks every day but not in other situations. Similarly, alternatives to plastic packaging are often heavier or more resource-intensive to produce ${ }^{5}$; therefore, labs should consider these factors and make informed choices before switching. When single-use plastics are an unavoidable byproduct of research output, they can sometimes be recycled through programmes offered by certain scientific suppliers (for examples, see REF. ${ }^{6}$ ). Ordering from vendors that offer these programmes is an important way to support them.

It may be difficult for individual lab members to prioritize addressing environmental issues if they are under pressure to produce research output, no matter the environmental cost. Therefore, lab heads have the important job of making clear that this is a priority and soliciting feedback from lab members about how best to implement it equitably. In labs that have the resources to hire lab managers or technicians, this can be part of the job description; in other cases, graduate students or postdocs could rotate responsibilities.

It is imperative that we adjust our research habits to confront the reality that is climate change. The programmes and behavioural changes we discuss here are certainly insufficient to address the whole extent of the environmental impact of our research programmes; however, many of the changes we argue for are nearly effortless and minimally affect (or even improve) research output, yet substantially reduce environmental impact and laboratory energy use. Therefore, we urge all research groups to evaluate their culture and develop a plan to address their environmental impact.

\section{Shepherd, A. et al. Mass balance of the Greenland Ice Sheet from 1992 to 2018. Nature 579, 233-239 (2020). \\ 2. The Louis Stokes Laboratories \& US National Renewable Energy Laboratory. Laboratories for the 21 st century: case studies. https:// www.nrel.gov/docs/fy02osti/30943.pdf (2001). \\ 3. Tedeschi, R. \& De Paoli, P. Collection and preservation of frozen microorganisms. Methods Mol. Biol. 675, 313-326 (2011). \\ 4. Hendrickson, C., Lave, L. \& Matthews, H. S. Environmental life cycle assessment of goods and services: An input-output approach (RFF Press, 2006). \\ 5. Franklin Associates, prepared for The Plastics Division of the American Chemistry Council. Life cycle impacts of plastic packaging compared to substitutes in the United States and Canada (2018). \\ 6. Kelesoglu, N. List of 10 life science lab suppliers with recycling services for end users. Labconcious https://www.labconscious.com/ green-lab-tips/2018/7/11/list-of-lab-suppliers-with-recycling- programs (2018)}

\section{Acknowledgements}

The authors thank the Harvard Green Labs Program, particularly Q. Gilly, for the invitation to participate in their initiatives and for their insights, as well as members of the Murthy Lab for their participation in the 'Shut the Sash' competition.

\section{Competing interests}

The authors declare no competing interests.

\section{RELATED LINKS}

Intergovernmental Panel on Climate Change: ipcc.ch

Harvard University Green Labs Program: green.harvard.edu/labs 